\title{
Laser assisted electron beam conditioning for free electron lasers
}

\author{
A. A. Zholents \\ Lawrence Berkeley National Laboratory, University of California, Berkeley, California 94720, USA
}

(Received 14 February 2005; published 17 May 2005)

\begin{abstract}
A new method for electron beam conditioning in free electron lasers is proposed. It uses the electron beam interaction with a laser light in two wiggler magnets separated by a strong-focusing channel. The effect of the conditioning is illustrated by the example of a hypothetical single-pass, high-gain free electron laser operating in the self-amplified spontaneous emission mode with the x-ray emission at $\lambda_{x}=$ $1.5 \AA$. The proposed conditioner is relatively compact and can be used as a practical add-on device to short $\mathrm{x}$-ray wavelength free electron lasers.
\end{abstract}

DOI: $10.1103 /$ PhysRevSTAB.8.050701

PACS numbers: $41.50 .+\mathrm{h}, 41.60 . \mathrm{Cr}, 42.55 . \mathrm{Vc}, 52.59 .-\mathrm{f}$

\section{INTRODUCTION}

In 1992, Sessler, Whittum, and Yu proposed a method of an electron beam conditioning [1] to improve the performance of free electron lasers (FELs). Since then, various conditioners (i.e., actual devices or schemes implementing conditioning in the electron beam) have been proposed [17]. Recently certain concerns about conditioners [1-7] were raised in [8] and were then addressed in [9]. Later two more conditioners were proposed $[10,11]$. However, conditioning of the electron beam for FELs producing radiation at few angstrom wavelengths, where conditioning is most needed to improve the performance of the FELs, remains a challenging task. Those FELs can benefit only from a large magnitude of conditioning which is well beyond the reach of the conditioners proposed so far. In this paper we propose a new conditioning method based on established technology which is capable of the large magnitude of conditioning that would be effective in the newly designed short x-ray wavelength FELs. In principle, such a conditioner can be used as an add-on device.

\section{METHOD}

A key provision for FEL operation is the so-called FEL resonance. Such resonance occurs when the longitudinal velocity of electrons in the electron beam is slowed down by the wiggling motion of an electron beam in the undulator and electrons slip behind the laser field (or selfemitted field) by one laser wave length after passing one undulator period. The FEL resonance allows for electrons to maintain interaction with the laser field in a stable phase over entire length of the undulator. But, electrons with different amplitudes of betatron oscillations and different energies have different longitudinal velocities and can not all be at the resonance at the same time. However, Sessler, Whittum, and Yu noticed that the betatron oscillations slow down the electrons longitudinal motion and propose to compensate it by providing a corresponding increase of electron energies effectively making them stay at the FEL resonance. By bringing more electrons into the resonance with the laser (or self-emitted) field, they expected better FEL performance, which was confirmed in numerical calculations (see, for example [1,9]). Strictly speaking they require the following "beam conditioning" [1]:

$$
\frac{\Delta \gamma}{\gamma_{C}}=C_{x} J_{x}+C_{y} J_{y}
$$

where $C_{r}$ is the conditioning parameter $(r \equiv x, y), \gamma_{C}$ is the relativistic factor, $J_{r}$ is the action $\left(\left\langle J_{r}\right\rangle \equiv \varepsilon_{r}\right)$, where $e_{r}$ is the geometrical rms emittance, and $\varepsilon_{N} \equiv \gamma_{C} \varepsilon_{r}$ is the normalized emittance. Assuming $\varepsilon_{y}=\varepsilon_{x}$ and equal focusing in both planes and following [9], we find a conditioning parameter that is needed for an FEL with undulator period $\lambda_{u}$ :

$$
C_{r} \equiv \frac{1}{2 \beta} \frac{\lambda_{u}}{\lambda_{x}}
$$

where $\lambda_{x}$ is the wavelength of the FEL emission, and $\beta$ is the average beta function in the undulator. Then, using Eq. (1), we write

$$
\Delta \gamma=C_{r} \varepsilon_{N} \frac{2 J_{r}}{\varepsilon_{r}} .
$$

In order to realize condition (3) in the electron beam, Vinokurov proposed the scheme shown in Fig. 1 [7] (see, also [8,9]). It consists of two rf linear accelerators and a strong-focusing beam line channel between them.

The first linac modulates the electron beam energy:

$$
\Delta \gamma_{1}=-\frac{e U}{m c^{2}} \sin \left(k_{\mathrm{rf}} z\right)
$$

where $k_{\mathrm{rf}}$ is the rf wave number, $z$ is the coordinate along the electron bunch $(z=0$ is at the center of the electron bunch), $e U=\Delta E_{\bmod }$ is the modulation amplitude, and $e$,

RF STRONG-FOCUSING CHANNEL RF

FIG. 1. A schematic of the Vinokurov's conditioner. 
$m$ are the electron charge and mass, $c$ is the speed of light, and $U$ is the rf voltage.

The strong-focusing channel provides particle delay:

$$
\begin{aligned}
\Delta z & =\frac{1}{2} \int\left(x^{\prime 2}+y^{\prime 2}\right) d s=2 \pi\left(\xi_{x} J_{x}+\xi_{y} J_{y}\right) \\
& =2 \pi \xi_{r} \frac{\varepsilon_{N}}{\gamma_{C}} \frac{2 J_{r}}{\varepsilon_{r}},
\end{aligned}
$$

where $s$ is the coordinate along the channel, $\xi_{x}, \xi_{y}$ are the chromaticity numbers for a channel and we assume $\xi_{x}=$ $\xi_{y}=\xi_{r}$. The Twiss functions of the channel are matched at the end of the channel to the values at the beginning of the channel. In order to avoid emittance growth, the chromatic Twiss functions must be matched and the following condition also must be satisfied [9]:

$$
W_{r}=\left[\left(\frac{\partial \alpha_{r}}{\partial E}\right)^{2}-2 \frac{\alpha_{r}}{\beta_{r}} \frac{\partial \alpha_{r}}{\partial E_{r}} \frac{\partial \beta_{r}}{\partial E}+\frac{\gamma_{r}}{\beta_{r}}\left(\frac{\partial \beta_{r}}{\partial E}\right)^{2}\right]^{1 / 2} \equiv 0,
$$

where $\alpha_{r}, \beta_{r}, \gamma_{r}$ are Twiss functions at the end of the channel.

The second linac removes the former energy modulation and leaves a residue energy offset that is correlated to the action

$$
\begin{aligned}
\Delta \gamma_{2} & =\frac{e U}{m c^{2}}\left\{-\sin \left(k_{\mathrm{rf}} z\right)+\sin \left[k_{\mathrm{rf}}(z-\Delta z)\right]\right\} \\
& \approx-2 \pi \xi_{r} k_{\mathrm{rf}} \frac{\Delta E_{\mathrm{mod}}}{\gamma_{C} m c^{2}} \cos \left(k_{\mathrm{rf}} z\right) \varepsilon_{N} \frac{2 J_{r}}{\varepsilon_{r}}
\end{aligned}
$$

where we assumed $k_{\mathrm{rf}} \Delta z<1$. Comparing (7) with (3), we define the conditioning parameter attainable with Vinokurov's conditioner at different $z$ locations within the electron bunch:

$$
C_{r}(z)=-2 \pi \xi_{r} k_{\mathrm{rf}} \frac{\Delta E_{\mathrm{mod}}}{\gamma_{C} m c^{2}} \cos \left(k_{\mathrm{rf}} z\right) .
$$

Typically, $k_{\mathrm{rf}} \sigma_{z} \ll 1$, where $\sigma_{z}$ is the electron bunch length, and we arrive at the same expression as in [9]:

$$
C_{r}(z) \equiv-2 \pi \xi_{r} k_{\mathrm{rf}} \frac{\Delta E_{\mathrm{mod}}}{\gamma_{C} m c^{2}} .
$$

Although Vinokurov's conditioner works in principle, the conditioning it produces is weak. In fact, if constrained by conventional linacs and focusing channel, it is several orders of magnitude too weak than what is needed [9] for a short wavelength $x$-ray FEL like the one described in [12].

In this paper we propose to substitute the rf linacs by lasers and wiggler magnets and increase the attainable conditioning parameter in Vinokurov's conditioner by several orders in magnitude. The new scheme is shown in Fig. 2. The laser pulse copropagates in the wiggler magnet at a small angle with the electron beam and produces energy modulation of the electrons at the laser wavelength $\lambda_{L}\left(k_{L}=2 \pi / \lambda_{L}\right.$ is the laser wave number). The electrons then go through a strong-focusing beam line channel and

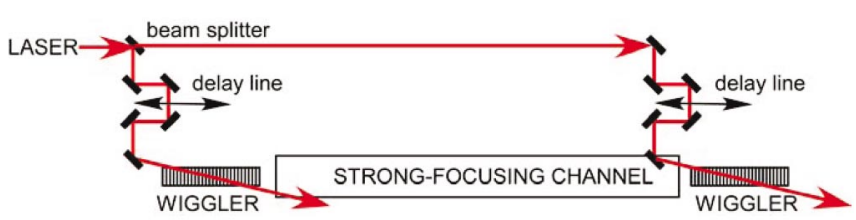

FIG. 2. (Color) A schematic of the laser assisted conditioner.

acquire time delays dependent upon betatron oscillations. Finally, the reverse modulation is applied via electron interaction with a second laser pulse in the second wiggler magnet. Arrival times of the electron beam and laser pulses in the wigglers are controlled by providing a tight synchronization between the laser pulses and an interferometric control of all path lengths with an active feedback.

In a new scheme we obtain for a conditioning parameter:

$$
C_{r}(z)=-2 \pi \xi_{r} k_{L} \frac{\Delta E_{\mathrm{mod}}}{\gamma_{C} m c^{2}} \cos \left(k_{L} z\right)
$$

where the only difference from the expression (8) consists in the appearance of $k_{L}$ instead of $k_{\mathrm{rf}}$. But, for all other parameters being equal, this implies a stronger conditioner by a huge factor $k_{L} / k_{\mathrm{rf}} \approx 10^{5}$.

However, now $k_{L} \sigma_{z} \gg 1$ and we have electrons at all phases with respect to the laser wave, i.e., electrons with a correct conditioning located near to $z= \pm n \lambda_{L}$ ( $n$ is the integer number), and electrons with incorrect conditioning (i.e., having a wrong sign of conditioning) located near to $z= \pm(n+0.5) \lambda_{L}$, and other electrons in between. The entire electron beam can now be viewed as a sequence of alternating slices of electrons with correct and incorrect conditioning. But, as long as the length of the slice, which is approximately equal to $\lambda_{L} / 2$, is longer than a slippage length of electrons in the FEL undulator, then the presence of two sorts of electrons and alternation of slices with correct and incorrect conditioning should not produce any other effect besides a trivial loss of coherent radiation from approximately one half of electrons.

\section{ILLUSTRATION OF THE METHOD}

We illustrate the proposed conditioner using a hypothetical single-pass, high-gain FEL operating in the selfamplified spontaneous emission (SASE) mode with x-ray emission at $\lambda_{x}=1.5 \AA$ produced by an electron beam with energy of $14 \mathrm{GeV}$. We consider an electron beam with emittance of $1.2 \mathrm{~mm}$-mrad, uncorrelated energy spread of $1.2 \mathrm{MeV}$, and peak current of $3.4 \mathrm{kA}$ and an undulator with $\lambda_{u}=3 \mathrm{~cm}$ and $\beta=20 \mathrm{~m}$. This choice of parameters is stimulated by the design report of the Linac Coherent Light Source [12].

Using (2) and (3) we calculate a required conditioning parameter $C_{r}=5 \times 10^{4} \mathrm{~cm}^{-1}$ and a required conditioning: $\Delta \gamma=6\left(J_{x}+J_{y}\right) / \varepsilon_{r}$. We choose to do conditioning at $1.5 \mathrm{GeV}$ electron energy. For energy modulation in the first 
wiggler we use the wiggler magnet with 10 periods and period length of $10 \mathrm{~cm}$ and a commercially available laser producing $\sim 100 \mathrm{fs}$ pulses with $6 \mathrm{~mJ}$ pulse energy at $\lambda_{L}=$ $800 \mathrm{~nm}$. For these parameters we calculate for energy modulation $\Delta E_{\text {mod }} \cong 30 \mathrm{MeV}$. Figure 3 shows energy distribution before and after the wiggler.

Then we assume that the electron beam will pass through a strong-focusing channel with $\xi_{r} \approx-5.3$. An example for such a channel is shown in Fig. 4. It consists of 16 FODO cells with $90^{\circ}$ betatron phase advance per cell in horizontal and vertical planes. Each quadrupole lens has a length of $25 \mathrm{~cm}$ and a magnetic field gradient of $4.3 \mathrm{kG} / \mathrm{cm}$. The left plot shows betatron functions and the right plot shows the $W_{r}$ chromatic functions defined in (6).

After passing through the channel, the electron beam proceeds into the second wiggler magnet. The second

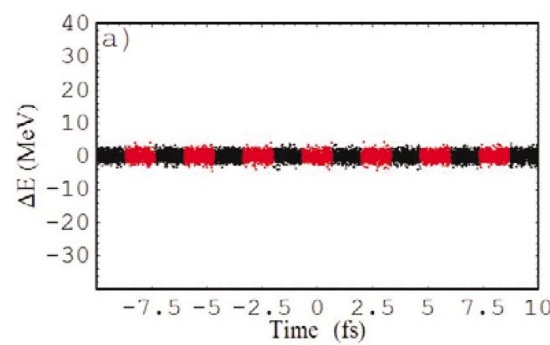

wiggler and the second laser pulse are identical to the first wiggler and the first laser pulse. The arrival time of a second laser pulse in the wiggler is adjusted relative to the arrival time of the electron bunch such as to produce energy modulation with a phase shift of $180^{\circ}$ with respect to the energy modulation in the first wiggler magnet. This modulation mostly cancels the energy modulation in the first wiggler magnet except for a "conditioning" part related to the electron path length delays in the FODO channel. Figure 5 demonstrates the effect of conditioning. Defining $\beta_{0 \|}=1-\lambda_{x} / \lambda_{u}$, we show there a distribution of electron longitudinal velocities:

$$
\Delta \beta_{\|}=\left\langle\beta_{\|}-\beta_{0 \|}\right\rangle=\frac{\lambda_{x}}{\lambda_{u}} \frac{2 \Delta \gamma}{\gamma_{C}}-\frac{2 J_{r}}{\beta}
$$

along the electron bunch averaged over the period of

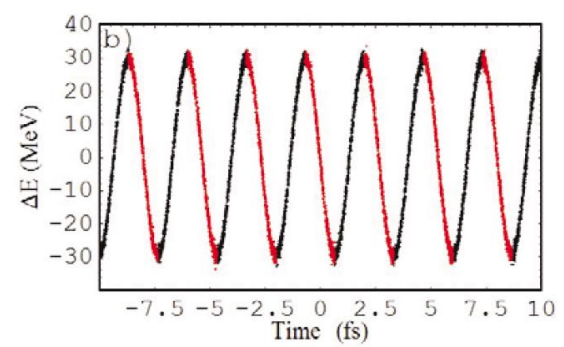

FIG. 3. (Color) The electron beam energy distribution before (a) and after (b) the wiggler. Color coded are equal length regions centered at $z= \pm n \lambda_{L}$ (red) and $z= \pm(n+0.5) \lambda_{L}$ (black). Only a short section of the electron beam is actually shown.
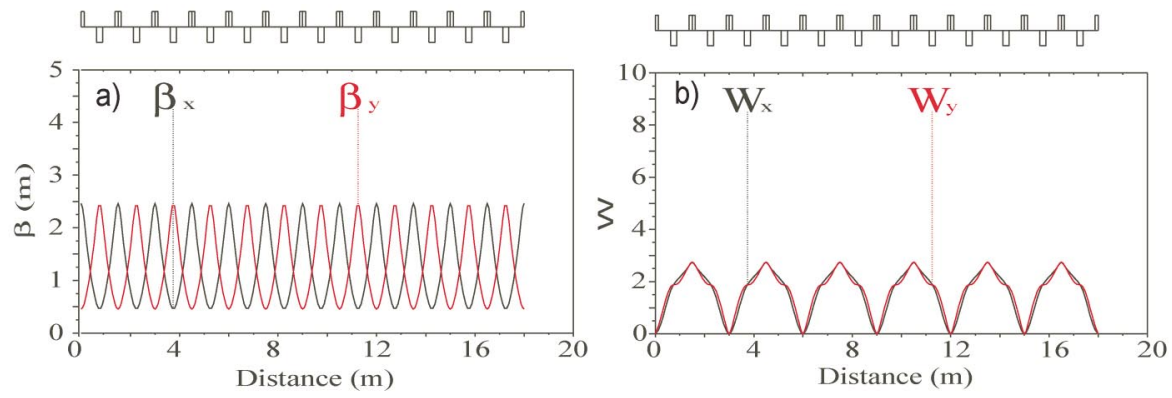

FIG. 4. (Color) The FODO channel with $\xi_{r}=-5.3$ (only 12 out 16 FODO cells are actually shown): (a) beta functions, (b) chromatic functions.
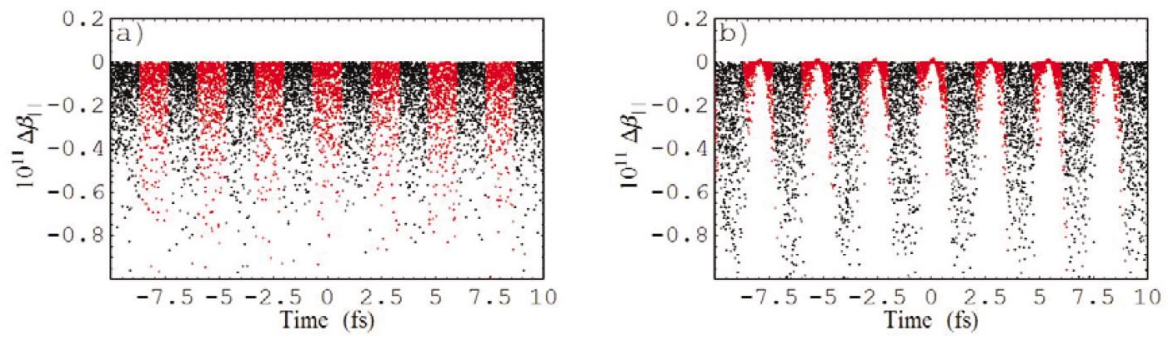

FIG. 5. (Color) A distribution of the electron longitudinal velocities for unconditioned (a) and conditioned (b) electron beams. An uncorrelated energy spread is ignored. Color coded sections within the electron beam are the same as in Fig. 3. 

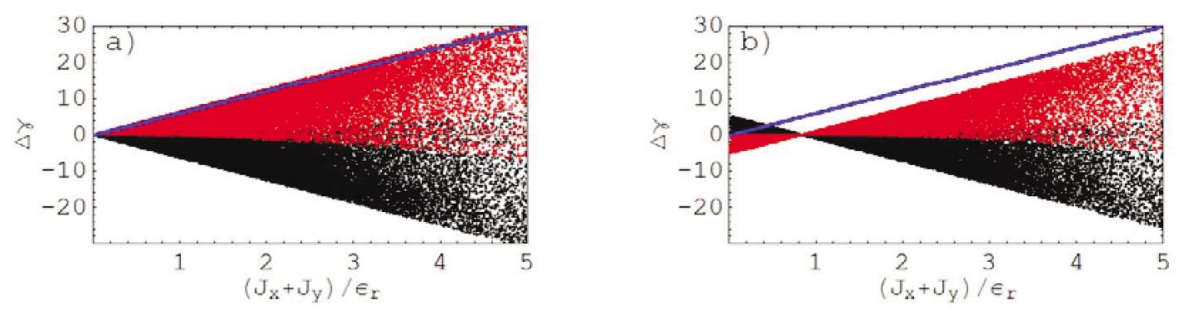

FIG. 6. (Color) Conditioning $\Delta \gamma$ versus $\left(J_{x}+J_{y}\right) / \varepsilon_{r}$ : (a) zero relative phase between two laser waves, (b) relative phase shift is $0.09 \mathrm{rad}$. The blue line corresponds to $\Delta \gamma=6\left(J_{x}+J_{y}\right) / \varepsilon_{r}$.
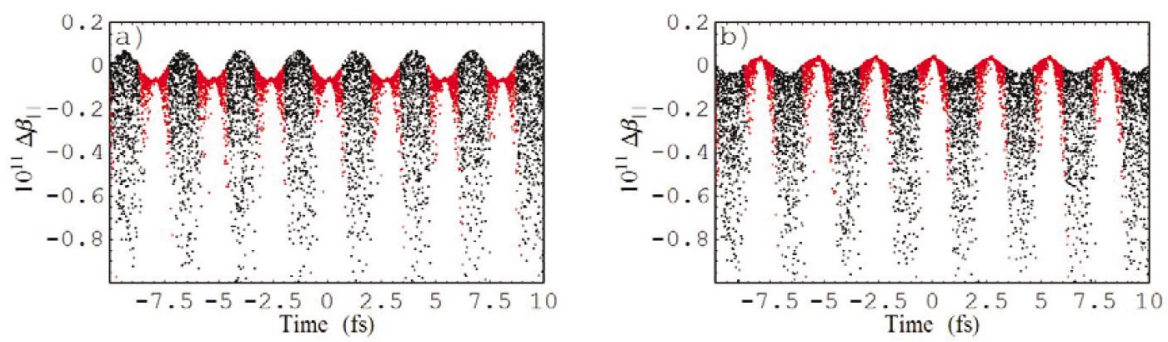

FIG. 7. (Color) A distribution of the electron longitudinal velocities in the case of the nonzero $\Delta \phi$ (see text): (a) $\Delta \phi=0.09$ rad, (b) $\Delta \phi=-0.03 \mathrm{rad}$.

betatron oscillation in the FEL undulator. Figure 5(a) shows a case of the electron beam without conditioning (for reference) and Fig. 5(b) shows the case of the electron beam with conditioning as prescribed above. In both cases we neglect the uncorrelated energy spread of the electron beam for clarity of the presentation (however, we use the actual energy spread in simulations described latter). As expected from Eq. (10), the amount of the conditioning varies along the electron bunch.

In Fig. 6(a) we plot $\Delta \gamma$ versus $\left(J_{x}+J_{y}\right) / \varepsilon_{r}$ for all electrons using same colors as in Fig. 3. There, we see that one half of the electrons leave the conditioner with a correct sign of $\Delta \gamma$ and one half of electrons leave the conditioner with an incorrect sign of $\Delta \gamma$. The spread of $\Delta \gamma$ for electrons with correct conditioning goes from zero up to the optimal value defined by a straight line $\Delta \gamma=$ $6\left(J_{x}+J_{y}\right) / \varepsilon_{r}$. In Fig. 6(b) we also show what happens if we shift the phase $\Delta \phi$ of the electron beam interaction with the second laser by $0.09 \mathrm{rad}$. Now zero spread in $\Delta \gamma$ appears at $\left(J_{x}+J_{y}\right) / \varepsilon_{r} \sim 1$. This setting produces a distribution of electron longitudinal velocities shown in Fig. 7(a). In Fig. 7 we plot $\Delta \beta_{\|}$for $\Delta \phi=0.09 \mathrm{rad}$ and $\Delta \phi=-0.03 \mathrm{rad}$. Based on these two plots and a plot in Fig. 5(b) we draw the conclusion that we need shot-to-shot fluctuations in $\Delta \phi$ to be less than $0.06 \mathrm{rad}$ and be centered at $\sim 0.03 \mathrm{rad}$. We note that $0.06 \mathrm{rad}$ at $800 \mathrm{~nm}$ wavelength corresponds to $\sim 8 \mathrm{~nm}$ in length. This number tells us how accurately we should control shot-to-shot variations of the electron beam path length between two wiggler magnets and two laser beam path lengths after the beam splitter. Evidently, it will require a high precision interferometric control of all path lengths with feedback and beam-based tuning. The main source of the path length jitter caused by the electron beam is a jitter of the electron beam trajectory at the entrance of the conditioner. We estimate that in order to comply with above requirement, a jitter of electron beam coordinate and angle should be less than 1 standard deviation of the correspondent beam sizes. Other sources that could cause variations in the path lengths are microphonics and temperature variations. Therefore, it helps to subject all path lengths to the same environment. We also studied how accurately we need to control the amplitude of the laser fields entering wiggler magnets and found that they should be equal to approximately $4 \%$ accuracy.

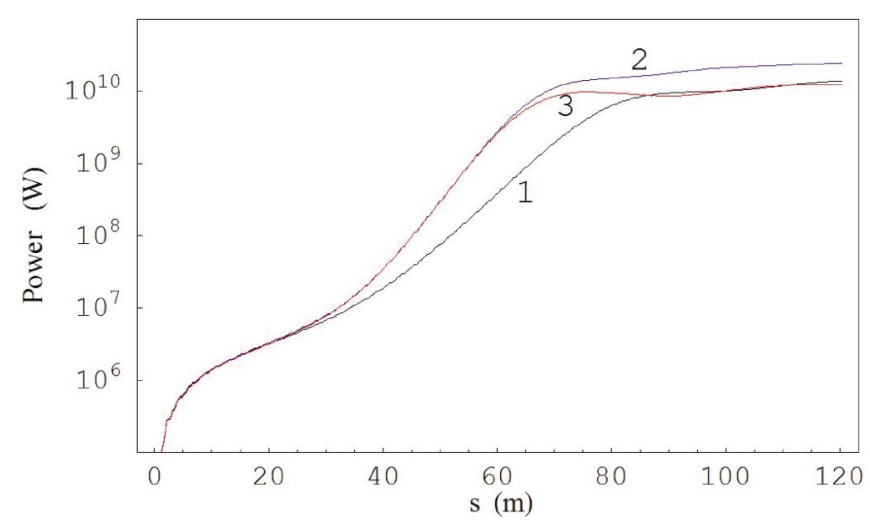

FIG. 8. (Color) The average $\mathrm{x}$-ray power versus distance for an electron beam with $1.2 \mathrm{~mm}$-mrad normalized emittance: 1 is the standard case without conditioning, 2 is the case of an ideal conditioning (see text for explanation), and 3 is the case of a practical conditioning with a proposed conditioner. 


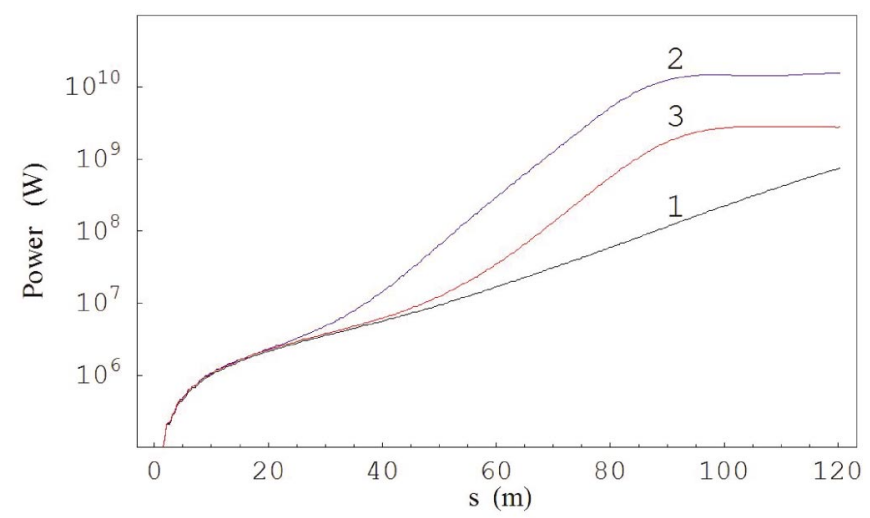

FIG. 9. (Color) The average x-ray power versus distance for an electron beam with $2.4 \mathrm{~mm}$-mrad normalized emittance: 1 is the case without conditioning, 2 is the case of an ideal conditioning, and 3 is the case of a practical conditioning with a proposed conditioner.

In the following discussion, we assume that a conditioned electron beam is accelerated to $14 \mathrm{GeV}$ and sent to an FEL undulator. We simulate the SASE process there using the FEL code GENESIS [13]. Results are shown in Figs. 8 and 9. Figure 8 shows the average x-ray power along the undulator calculated for a normalized electron beam emittance of $1.2 \mathrm{~mm}$-mrad. In this plot, black curve 1 corresponds to a standard case without conditioning, blue curve 2 to a case of an ideal conditioning without time dependence, i.e., without cosine function in (10), and red curve 3 corresponds to a practical case with an electron beam conditioned in the above described conditioner. We see that conditioning gives shorter saturation length and that a saturated power in the practical case is a few times smaller than in the ideal case. Figure 9 shows results of similar calculations performed for a normalized electron beam emittance of $2.4 \mathrm{~mm}-\mathrm{mrad}$, i.e., twice the nominal value. The definition of curves there is the same as in Fig. 8. Here we see that conditioning helps to reach saturation in a less than $100 \mathrm{~m}$ long undulator while without conditioning saturation would require a much longer undulator. For an even better result one can try to use a smaller average beta function in the undulator as was recommended in [9], but it will require stronger conditioning which may not be easily achievable.

\section{ACKNOWLEDGMENTS}

This work was supported by the Director, Office of Science, of the U.S. Department of Energy, under Contract No. DE-AC03-76SF00098. Useful discussions with A. Wolski, W. Fawley, G. Penn, A. Sessler, and J. Wurtele are gratefully acknowledged.

[1] A. M. Sessler, D. H. Whittum, and L.-H. Yu, Phys. Rev. Lett. 68, 309 (1992).

[2] P. Sprangle, B. Hafizi, G. Joyce, and P. Serafim, Nucl. Instrum. Methods Phys. Res., Sect. A 331, 6 (1993).

[3] G. R. Neil and H. Liu, in Proceedings of the Particle Acceleration Conference, Washington, DC, 1993 (IEEE, Piscataway, NJ, 1993), p. 1563.

[4] H. Liu and G. R. Neil, in Proceedings of the Particle Acceleration Conference, Washington, DC, 1993 (Ref. [3]), p. 279.

[5] V. A. Papadchiev, Nucl. Instrum. Methods Phys. Res., Sect. A 358, ABS79 (1995).

[6] V. A. Papadchiev, Nucl. Instrum. Methods Phys. Res., Sect. A 375, 132 (1996).

[7] N. A. Vinokurov, Nucl. Instrum. Methods Phys. Res., Sect. A 375, 264 (1996).

[8] P. Emma and G. Stupakov, Phys. Rev. ST Accel. Beams 6, 030701 (2003).

[9] A. Wolski, G. Penn, A. Sessler, and J. Wurtele, Phys. Rev. ST Accel. Beams 7, 080701 (2004).

[10] P. Emma and G. Stupakov, in Proceedings of the 2004 European Particle Acceleration Conference, p. 503, http: // accelconf. web. cern.ch / accelconf / e04 / PAPERS/ MOPKF080.PDF

[11] C. B. Schroeder, E. Esarey, and W. P. Leemans, Phys. Rev. Lett. 93, 194801 (2004).

[12] See, e.g., Linac Coherent Light Source Design Study Report, Report No. SLAC-R-521, 1998.

[13] S. Reiche, Nucl. Instrum. Methods Phys. Res., Sect. A 429, 243 (1999). 